\title{
A near-infrared fluorescent probe for selective detection of fluorion
}

\author{
Yan-Fei Kang1, ${ }^{1,}$ Fan Wei ${ }^{3}$, Ya-Li Meng1, ${ }^{1,}$, Zhen-Hui Xin ${ }^{1,2}$, Dong Wei ${ }^{1, ~ * ~}$ \\ ${ }^{1}$ Hebei North University, Hebei Key Laboratory of Quality \& Safety Analysis-Testing for Agro-Products and Food, 11 Dia- \\ mond Street South, Zhangjiakou, 075000, Hebei Province, People's Republic of China \\ ${ }^{2}$ Hebei North University, College of Laboratory Medicine, 11 Diamond Street South, Zhangjiakou, 075000, Hebei Pro- \\ vince, People's Republic of China \\ ${ }^{3}$ Northeast Agricultural University, College of Food Science, 600 Changjiang Street, Xiangfang District, 150030, HarBin, \\ China \\ ${ }^{*}$ Corresponding author: e-mail: zjkzdsyswd@163.com
}

\begin{abstract}
In this work, we have designed and synthesized the fluorescent probe 1, which was capable to selectively detect fluoride anion $\left(\mathrm{F}^{-}\right)$. More importantly, the probe 1 possessed near-infrared excitation and emission wavelengths (excitation at $650 \mathrm{~nm}$ and emission at 695), and the probe solution had changed dramatically from yellow to cyan with the addition of $\mathrm{F}^{-}$. In addition, the fluorescence intensity exhibited perfectly positive correlation with concentration of $\mathrm{F}^{-}$concentration from 0 to $40 \mu \mathrm{M}\left(\mathrm{R}^{2}=0.9972\right)$, which offered the important condition for quantitative analysis. The probe 1 owned detection limit of $46 \mathrm{nM}$. Therefore, this near-infrared probe can be of great benefit for detecting $\mathrm{F}^{-}$in practical application.
\end{abstract}

Keywords: Probe, Fluorescence, Fluoride anion, Selectivity, Near-infrared.

\section{INTRODUCTION}

Fluoride ion $\left(\mathrm{F}^{-}\right)$is the smallest anion, which is indispensable trace element in the human body and plays critical roles in a variety of biological processes ${ }^{1,2}$. Low levels of $\mathrm{F}^{-}$intake are closely connected with risk of decayed tooth and osteoporosis ${ }^{3}$. However, excessive $\mathrm{F}^{-}$ingestion easily gave rise to many diseases that are, skeletal fluorosis, kidney failure, or urolithiasis, even cancer ${ }^{4}$. In addition, $\mathrm{F}^{-}$contamination in soil and water affect plant growth and development. $\mathrm{F}^{-}$is also abundant in many industrial workplaces, which can be used as oxidizer in rocket fuel burn, in nuclear fuel, in computer chips and microelectronic sensors, and television screens ${ }^{5}$. Therefore, the accurate determination of fluoride is of growing importance in both environmental and biological systems. The conventional assay methods of fluoride commonly include ion-selective electrode, colorimetry, capillary electrophoresis and so on ${ }^{6,7}$. Among them, fluorescence detection techniques have attracted much attention based on operational simplicity, high sensitivity, and cell bioimaging ${ }^{8}$.

Up to now, although a lot of fluorescent probes have been developed to detect $\mathrm{F}^{-9-13}$, they still have some limitations more or less in practical application. Poor selectivity and sensitivity is the common problem for some probes. Moreover, most probes have short-wavelength excitation and emission spectra, which increase difficulty for detecting $\mathrm{F}^{-}$in the biological systems because of poor anti-interference ability. Meanwhile, light in the short-wavelength region has limited tissue penetration and it is easily scattered and absorbed by biomolecules. Therefore, in order to improve this drawback, the fluorescent probe should be constructed with excitation and emission wavelengths in the near-infrared.

Therefore, in this work, we designed and synthesized the fluorescence probe 1 ((E)-2-(3-(4-((tert-butyldiphenylsilyl)oxy)styryl)-5,5-dimethylcyclohex-2-en-1-ylidene) malononitrile) and envisioned the probe can selectively detect $\mathrm{F}^{-}$with the near-infrared excitation and emission spectra. The probe 1 was designed on the base of internal charge transfer (ICT) mechanism ${ }^{14}$ with 2-(3,5,5-trime- thylcyclohex-2-en-1-ylidene)malononitrile and phenol derivative as electron-withdrawing group and a potential strong electron-donating group, respectively. In addition, we chose a conventional tert-butyldiphenylsilyl (TBDPS) moiety as the specific recognition group for $\mathrm{F}^{-1}$. The results exhibited the probe $\mathbf{1}$ can selectively detect $\mathrm{F}^{-}$ with excitation wavelength at $650 \mathrm{~nm}$.

\section{EXPERIMENTAL}

\section{Materials and apparatus}

${ }^{1} \mathrm{H} /{ }^{13} \mathrm{C}$ NMR spectra were recorded on a Bruker AVIII-400/600 MHz spectrometer. High resolution mass spectra (HRMS) were measured with Thermo (orbitrap Elite). Absorption spectra were measured using a Thermo (BioMate 3S) UV/Vis spectrophotometer. Fluorescence measurements were carried out with a F97pro fluorospectrophotometer. Unless otherwise noted, materials were purchased from commercial suppliers and used without further purification. All the solvents were purified and dried according to general methods.

\section{Synthesis of probe 1 (Scheme 1)}

2-(3,5,5-Trimethylcyclohex-2-en-1-ylidene)malononitrile (1) isophorone $(6.902 \mathrm{~g}, 50 \mathrm{mmol})$ and malononitrile (3.3 g, $50 \mathrm{mmol}$ ) were dissolved in $250 \mathrm{~mL}$ round bottomed flask with absolute ethanol as solvent. Subsequently, the piperidine $(0.425 \mathrm{~g}, 5 \mathrm{mmol})$ was dropwise added. The reaction was refluxed for $8 \mathrm{~h}$ and then the mixture was cooled and poured into ice water. Lastly, the sediment was collected and recrystallized by ethanol. The compound 1 was obtained as a white crystal $(3.961 \mathrm{~g}$, 43\%). ${ }^{1} \mathrm{H}-\mathrm{NMR}\left(600 \mathrm{MHz}, \mathrm{CCl}_{3} \mathrm{D}\right): \delta=1.01(\mathrm{~s}, 6 \mathrm{H})$, $2.03(\mathrm{~s}, 3 \mathrm{H}), 2.17(\mathrm{~s}, 2 \mathrm{H}), 2.51(\mathrm{~s}, 2 \mathrm{H}), 6.61(\mathrm{~s}, 1 \mathrm{H})$ ppm; ${ }^{13}$ C-NMR (125 MHz, $\left.\mathrm{CCl}_{3} \mathrm{D}\right): \delta=25.4,28.0,32.5$, $42.8,45.8,78.5,112.5,113.3,120.7,159.8,170.5$ ppm.

(E)-2-(3-(4-Hydroxystyryl)-5,5-dimethylcyclohex-2-en1-ylidene)malononitrile (2) 2-(3,5,5-trimethylcyclohex-2-en-1-ylidene)malononitrile $(0.9313 \mathrm{~g}, 5 \mathrm{mmol})$ and p-hydroxy benzaldehyde $(0.6108 \mathrm{~g}, 3 \mathrm{mmol})$ were dissolved in $100 \mathrm{~mL}$ round bottomed flask with $50 \mathrm{~mL}$ of 
acetonitrile as solvent and piperidine $(10 \mathrm{~d})$ was added dropwise . The solution was refluxed for $8 \mathrm{~h}$. The mixture was washed with water, and extracted with ethyl acetate and dried by sodium sulfate. The solvent was evaporated and the residue was purified by flash column chromatography (dichloromethane / methyl alcohol $100 / 1$ ) to obtained the red compound $2(1.0636 \mathrm{~g}, 73 \%) .{ }^{1} \mathbf{H}-\mathbf{N M R}$ $\left(400 \mathrm{MHz}, \mathrm{DMSO}-d_{6}\right): \delta=1.01(\mathrm{~s}, 6 \mathrm{H}), 2.53(\mathrm{~s}, 2 \mathrm{H})$, 2.59 (s, $2 \mathrm{H}), 6.78-6.80(\mathrm{~m}, 3 \mathrm{H}), 7.18(\mathrm{~d}, J=16.2 \mathrm{~Hz}$, $1 \mathrm{H}), 7.23(\mathrm{~d}, J=16.2 \mathrm{~Hz}, 1 \mathrm{H}), 7.55(\mathrm{~d}, J=8.4 \mathrm{~Hz}$, $1 \mathrm{H}), 9.98$ (s, $1 \mathrm{H})$ ppm; ${ }^{13} \mathrm{C}-\mathbf{N M R}(100 \mathrm{MHz}, \mathrm{DMSO}-$ $\left.d_{6}\right): \delta=27.5,31.7,38.2,42.3,74.8,113.3,114.2,115.9$, 121.4, 126.3, 127.1, 129.9, 138.3, 156.8, 159.3, 170.3 ppm.

(E)-2-(3-(4-((tert-butyldiphenylsilyl)oxy) styryl)-5,5-dimethylcyclohex-2-en-1-ylidene)malononitrile (Probe 1) To a solution of compound $2(0.40 \mathrm{~g}, 1.4 \mathrm{mmol})$ in anhydrous DMF (20 mL), diisopropylethylamine $(0.26$ $\mathrm{g}, 2.1 \mathrm{mmol})$ were added and the mixture was stirred at room temperature for $15 \mathrm{~min}$. then the diphenyl tert-butyl chlorosilane $(0.58 \mathrm{~g}, 2.1 \mathrm{mmol})$ was added and the reaction continued to stir for $4 \mathrm{~h}$ under room temperature. Lastly, the mixture was quenched with water, and extracted with ethyl acetate. The combined organic layers were dried by sodium sulfate and purified by flash column chromatography (petroleum ether / ethyl acetate $10 / 1)$ to obtained the product (probe 1) $(0.075 \mathrm{~g}, 10 \%)$. ${ }^{1} \mathbf{H}-\mathbf{N M R}\left(400 \mathrm{MHz}, \mathrm{CCl}_{3} \mathrm{D}\right): \delta=1.05(\mathrm{~s}, 2 \mathrm{H}), 1.07(\mathrm{~s}$, $15 \mathrm{H}), 1.11(\mathrm{~s}, 2 \mathrm{H}), 7.36-7.42(\mathrm{~m}, 10 \mathrm{H}), 7.7-7.73(\mathrm{~m}$, 7H) ppm; ${ }^{13} \mathbf{C}$-NMR (100 MHz, $\left.\mathrm{CCl}_{3} \mathrm{D}\right): \delta=19.2,19.6$, 26.6, 26.7, 28.1, 32.1, 39.3, 43.1, 120.6, 122.8, 127.2, 127.9, 128.0, 128.9, 129.1, 129.8, 130.2, 132.5, 134.9, 135.3, 135.6, 137.1, 154.4, 157.5, 169.4 ppm; HRMS (ESI) m/z calcd for $\mathrm{C}_{35} \mathrm{H}_{36} \mathrm{~N}_{2} \mathrm{OSi}(\mathrm{M}+\mathrm{Na})$ : 551.2489. Found: 551.2463, error: $4.7 \mathrm{ppm}$.

\section{Bioimaging of probe 1 in $\mathrm{MCF}-7$ cells}

MCF-7 cells $\left(5 \times 10^{4} / \mathrm{mL}\right)$ were seeded in 6-well flat microtiter plates for adherence for $24 \mathrm{~h}$. The control group. Cells were incubated with probe $1(20 \mu \mathrm{M})$ for 30 min, and then washed with PBS for 3 times. The experiment group. The cells were pre-incubated with probe $\mathbf{1}$ $(20 \mu \mathrm{M})$ for $30 \mathrm{~min}$ and subsequently incubated by the appointed concentration of $\mathrm{F}^{-}$for another $30 \mathrm{~min}$, and then washed with PBS for 3 times. Fluorescence images of probe $\mathbf{1}$ were got by a fluorescence microscope.

\section{RESULTS AND DISSCUSSION}

We synthesized the probe $\mathbf{1}$ and the synthetic route is shown in Scheme 1. First, we synthesized the (2-(3,5,5-trimethylcyclohex-2-en-1-ylidene)malononitrile) (Compound 1) according to relevant references ${ }^{\mathbf{1 5}}$. Subsequently, compound 1 reacted with $p$-hydroxy benzaldehyde to give the compound 2 by Aldol reaction. Lastly, the compo-

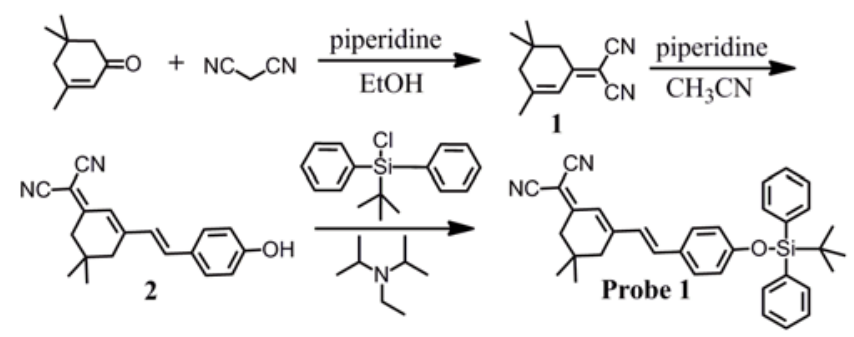

Scheme 1. Synthesis of probe 1 und 2 was further reacted with diphenyl tert-butylchlorosilane to obtain the probe $\mathbf{1}$ under basic condition in $3 \%$ all yield and the probe structure was confirmed by NMR and HRMS (ESI).

As the probe $\mathbf{1}$ was obtained, we next investigate its optical properties. Firstly, the absorption bands of probe $\mathbf{1}$ and probe 1 toward $\mathrm{F}^{-}$were surveyed in the solution $(\mathrm{THF} / \mathrm{DMSO}=1: 1, \mathrm{v} / \mathrm{v})$. As shown in Fig. 1 , probe $\mathbf{1}$ showed an absorption band at $425 \mathrm{~nm}$. Upon the addition of $\mathrm{F}^{-}$, the peak of absorption band red shifted to $675 \mathrm{~nm}$ and the new absorption peak increased gradually with increasement of reaction time from 1 to $5 \mathrm{~min}$ at room temperature. In order to avoid the influence of excitation light for emission spectrum, we choose the $650 \mathrm{~nm}$ as excitation wavelength. More interestingly, the solution of probe 1 changed from yellow to cyan with the addition of $\mathrm{F}^{-}$. Hence, this result showed that probe $\mathbf{1}$ can identify and detect $\mathrm{F}^{-}$by "naked-eye" colorimetric change, which provided convenience for practical application.

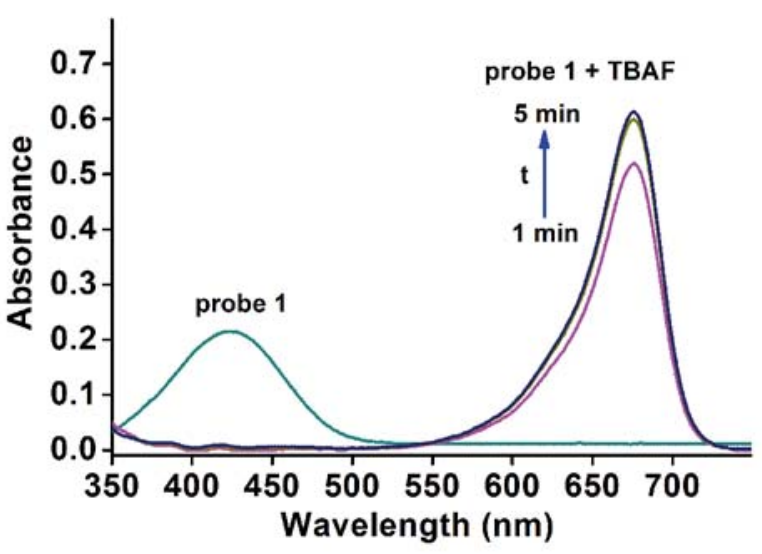

Figure 1. The UV-vis absorption spectra of probe 1 and probe 1 toward fluorion

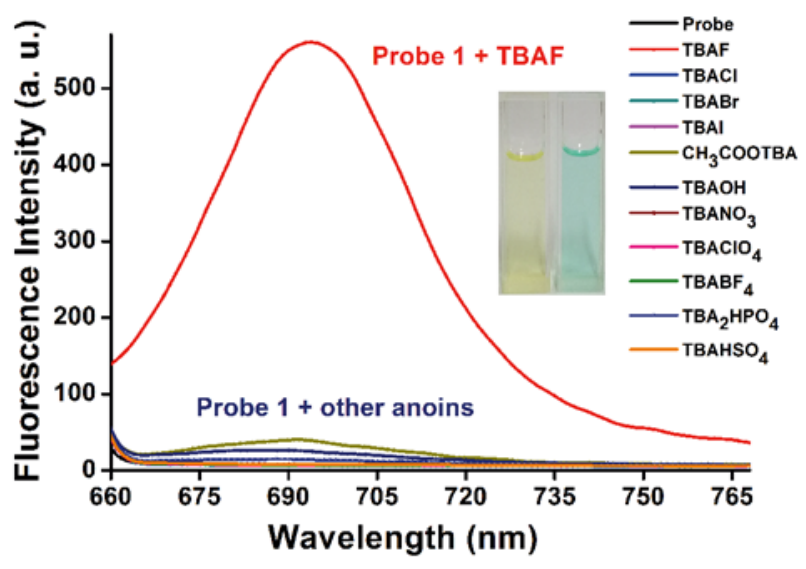

Figure 2. The fluorescence spectra of probe 1 in the presence of different anions. inset: The color change of probe 1 before and after addition of fluorine ion

To investigate the selectivity of probe 1 toward $\mathrm{F}^{-}$, we screened various anions (TBA salts of $\mathrm{HSO}_{4}{ }^{-}, \mathrm{ClO}_{4}{ }^{-}$, $\mathrm{CH}_{3} \mathrm{COO}^{-}, \mathrm{BF}_{4}^{-}, \mathrm{NO}_{3}{ }^{-}, \mathrm{HPO}_{4}{ }^{2-}, \mathrm{I}^{-}, \mathrm{Br}^{-}, \mathrm{Cl}^{-}, \mathrm{F}^{-}, \mathrm{OH}^{-}$, $100 \mu \mathrm{M})$ (Fig. 2). The fluorescence intensity increased markedly at $695 \mathrm{~nm}$, when the probe $1(20 \mu \mathrm{M})$ was incubated by $\mathrm{F}^{-}$for $2 \mathrm{~min}$ in solution (THF/DMSO = $1: 1, \mathrm{v} / \mathrm{v})$ at room temperature with excitation wavelength at $650 \mathrm{~nm}$. As we all known, the excitation and emission wavelength was longer than that of most pre- 
vious reports ${ }^{11-13}$. More interestingly, other anions did not cause significant increase of fluorescence intensity, even change induced by hydroxyl ion also was negligible. Moreover, the relationship between fluorescence intensity and concentration was quantitatively analyzed by titration (Fig. 3a). Results exhibited the satisfactory positive correlation between fluorescence intensity and concentration of $\mathrm{F}^{-}$from 0 to $40 \mu \mathrm{M}\left(\mathrm{R}^{2}=0.9972\right)$ (Fig. 3b), which is an advantage condition for quantitative analysis of $\mathrm{F}^{-}$.

Meanwhile, we further finished the competition experiment to survey interference of coexisting ions for detecting $\mathrm{F}^{-}$(Fig. 4a). The results exhibited the fluorescence intensity change of probe $\mathbf{1}$ toward $\mathrm{F}^{-}$is insignificant in the presence of other anions. Therefore, these results displayed probe $\mathbf{1}$ can selectively detect $\mathrm{F}^{-}$. On the other hand, we investigated the fluorescence intensity change of probe toward $\mathrm{F}^{-}$with incubation time increase (Fig. 4b). The reaction was completed after the probe was incubated by $\mathrm{F}^{-}$for 2 min and the fluorescence intensity hardly decayed within 20 minutes. Furthermore, the probe itself did not cause a change in fluorescence intensity with the reaction time extension. Thus, these results provided the condition for practical application. Moreover, the detection limit of the probe was determined $(3 \sigma / \mathrm{k})$ to be $46 \mathrm{nM}$.

a)

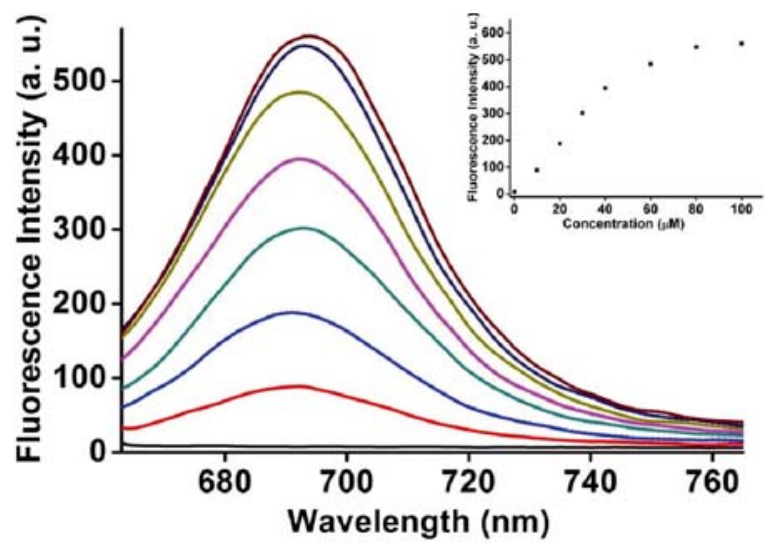

b)

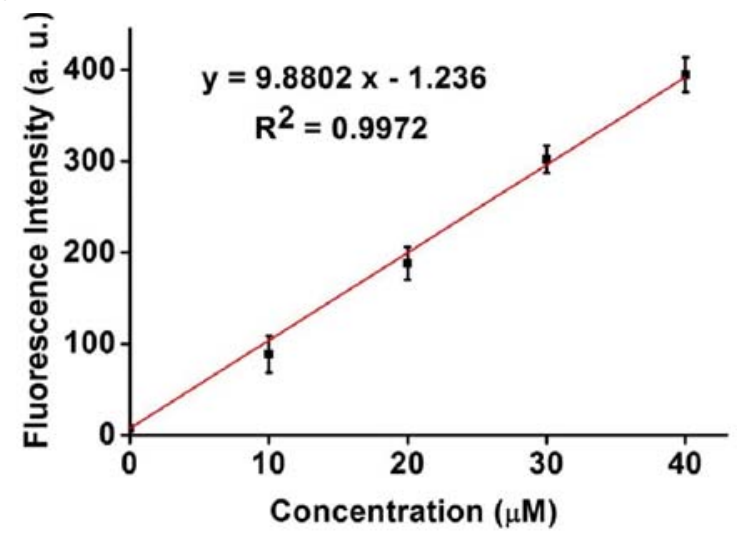

Figure 3. (a) The fluorescence intensity response of probe 1 for the various concentration of $\mathrm{F}^{-}$in solution $(\mathrm{THF} / \mathrm{DMSO}=1: 1, \mathrm{v} / \mathrm{v})$ at room temperature with excitation wavelength at $650 \mathrm{~nm}$. (b) The linear relationship between fluorescence intensity and $\mathrm{F}^{-}$ concentration
To study the mechanism, we test the absorption of compound 2 in the same condition (THF/DMSO $=1: 1, \mathrm{v} / \mathrm{v}$ ) (Fig. 5). The result displayed compound 2 have absorption peak at $437 \mathrm{~nm}$. However, with addition of TBAF, the absorption peak of compound 2 was red shifted to $675 \mathrm{~nm}$, which was consistent with the absorption peak of probe $\mathbf{1}$ toward $\mathrm{F}^{-}$. Therefore, we proposed possible mechanism in the Fig. 6 . The probe with tert-butyldiphenylsilyl (TBDPS) moiety identifies the fluoride ion by Si-O band breakage, which had been proved in many previous reports ${ }^{9-13}$. The compound 2 can be obtained with reaction of probe 1 and $\mathrm{F}^{-}$. Based on the basic conditions provided by TBAF, the compound 2 further loses the hydrogen proton to lead absorption red shift and the turn on the fluorescence ${ }^{\mathbf{1 6}}$.

To explore the practical application, we inspected the bioimaging of probe $\mathbf{1}$ in the living cells. Firstly, we measured the cytotoxicity of the probe in $\mathrm{MCF}-7$ cells by MTT method (Fig. 7). The results displayed the survival rate did not significantly change with the addition of $80 \mu \mathrm{M}$ probe. Therefore, the probe can be used in the biosystem. Subsequently, we executed the cell imaging experiment in MCF-7 cells (Fig. 8). The results showed the blue fluorescence markedly appeared when the cells was pre-incubated with $30 \mathrm{~min}$ and then continued to incubate with $\mathrm{F}^{-}$for $30 \mathrm{~min}$. Moreover, the fluorescence intensity was increased clearly along with increase of TBAF concentration from 100 to $500 \mu \mathrm{M}$.

a)

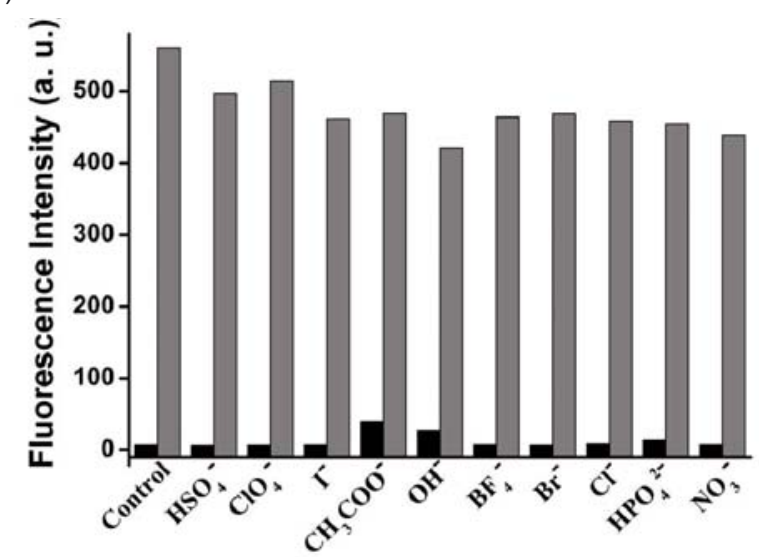

b)

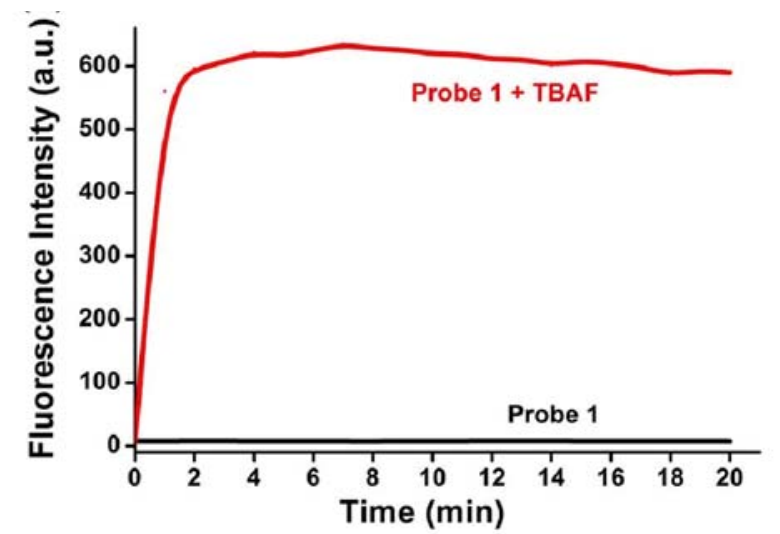

Figure 4. (a) Fluorescence intensity change of probe $1(20 \mu \mathrm{M})$ toward $\mathrm{F}^{-}$in the presence of various analytes in solution $(\mathrm{THF} / \mathrm{DMSO}=1: 1, \mathrm{v} / \mathrm{v})$ at room temperature with excitation wavelength at $650 \mathrm{~nm}$. Black bar: probe $1+$ analytes; Grey bar: probe $1+\mathrm{F}^{-}+$analytes; (b) The time-dependent fluorescence intensity response of probe 1 in the presence/absence of TBAF 


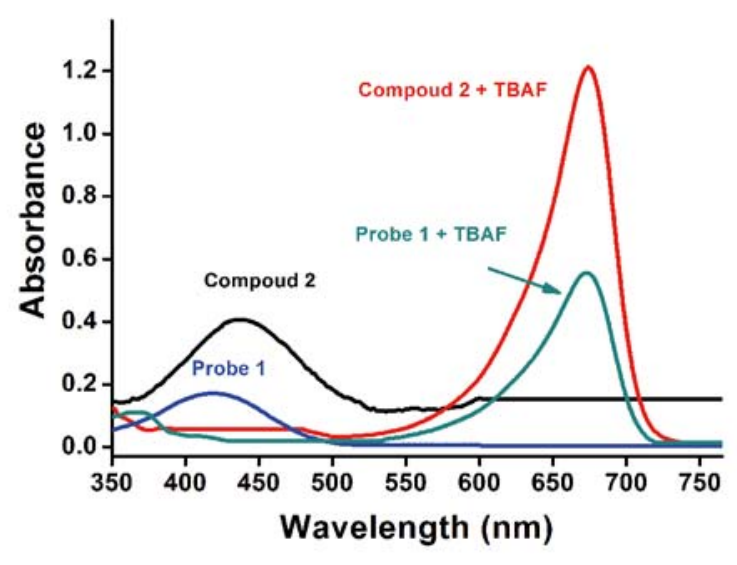

Figure 5. The UV-vis absorption spectra of compounds

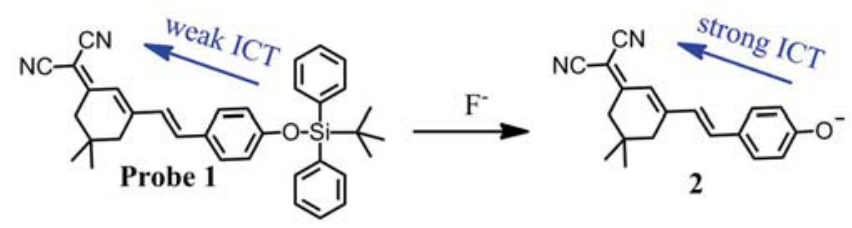

Figure 6. The possible mechanism between of probe 1 and $\mathrm{F}^{-}$

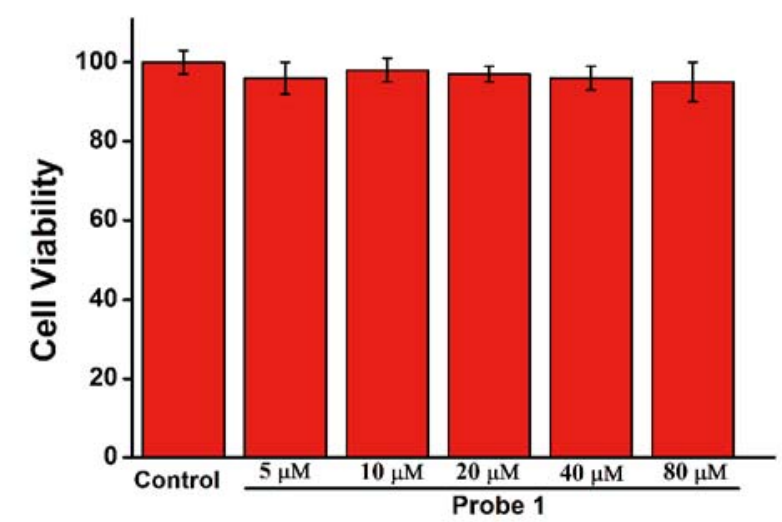

Figure 7. The cytotoxicity of probe 1 in MCF-7 cells

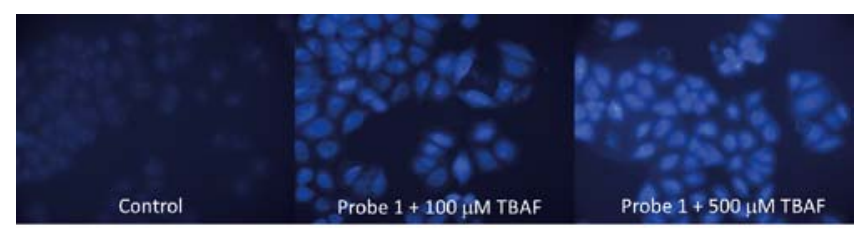

Figure 8. Fluorescence Imaging of probe $1(20 \mu \mathrm{M})$ in the MCF-7 cells

\section{CONCLUSIONS}

In summary, we have developed the fluorescent probe 1, which exhibited a highly selective and sensitive response to $\mathrm{F}^{-}$in the test. When the $\mathrm{F}^{-}$was added, the fluorescence intensity enhanced obviously with the color change from yellow to cyan by "naked-eye" within $2 \mathrm{~min}$. Therefore, the probe 1 could selectively detect $\mathrm{F}^{-}$. Furthermore, the probe 1 possessed near-infrared excitation and emission wavelengths (excitation at 650 $\mathrm{nm}$ and emission at 695), which offered the favorable condition for anti-interference and penetration. In addition, the fluorescence intensity exhibited perfectly positive correlation with concentration of $\mathrm{F}^{-}$concentration from 0 to $40 \mu \mathrm{M}\left(\mathrm{R}^{2}=0.9972\right)$. Therefore, we believe this near-infrared probe can be of great benefit for detecting $\mathrm{F}^{-}$in practical application.

\section{ACKNOWLEDGEMENTS}

This work was supported by the Natural Science Foundation of Hebei Province (No. B2016405026), Young Elitist Foundation of Hebei Province (No. BJ2016003), Hebei North University PhD startup fund (No. 12995556) and Hebei North University Fund (No. YB2018002; No. QN2018008; No. QN2018012).

\section{CONFLICT OF INTEREST}

The authors declare no conflicts of interest.

\section{LITERATURE CITED}

1. Zhang, S., Fan, J., Zhang, S., Wang, J., Wang, X., Du, J. \& Peng, X. (2014). Lighting up fluoride ions in cellular mitochondria using a highly selective and sensitive fluorescent probe. Chem. Commun. 50, 14021-14024. DOI: 10.1039/c4cc05094k.

2. Li, Y., Duan, Y., Zheng, J., Li, J., Zhao, W., Yang, S. \& Yang, R. (2013). SelF-assembly of graphene oxide with a silyl-appended spiropyran dye for rapid and sensitive colorimetric detection of fluoride ions. Anal. Chem. 85, 11456-11463. DOI: 10.1021/ac402592c.

3. Zeng, L., Yuan, Y., Jiang, C., Mu, J., Li, F., Wan, Y., Xu, H., Qu, J., Huang, P. \& Lin, J. (2019). A near-infrared turn-on probe for in vivo chemoselective photoacoustic detection of fluoride ion. Dyes and Pigments 165, 408-414. DOI: 10.1016/j. dyepig.2019.02.049.

4. Duke, R.M., Veale, E.B., Pfeffer, F.M., Kruger, P.E. \& Gunnlaugsson, T. (2010). Colorimetric and fluorescent anion sensors: an overview of recent developments in the use of 1,8-naphthalimide-based chemosensors. Chem. Soc. Rev. 39, 3936-3953. DOI: 10.1039/B910560N.

5. Dichiarante,V., Milani, R. \& Metrangolo, P. (2018). Natural surfactants towards a more sustainable fluorine chemistry, Green Chem. 20, 13-27. DOI: org/10.1039/C7GC03081A.

6. Wang, C.Y., Yang, S., Yi, M., Liu, C.H., Wang, Y.J., Li, J.S., Li, Y.H. \& Yang, R.H. (2014). Graphene oxide assisted fluorescent chemodosimeter for high-performance sensing and bioimaging of fluoride ions. ACS Appl. Mater. Interfaces 6, 9768-9775. DOI: 10.1021/am502142d.

7. Zhou, Y., Zhang, J.F. \& Yoon, J. (2014). Fluorescence and colorimetric chemosensors for fluoride-ion detection. Chem. Rev. 114, 5511-5571. DOI: 10.1021/cr400352m.

8. Urano, Y. (2012). Novel live imaging techniques of cellular functions and in vivo tumors based on precise design of small molecule-based 'Activatable' fluorescence probes. Curr. Opin. Chem. Biol. 16, 602-608. DOI: 10.1016/j.cbpa.2012.10.023

9. Turan, I.S., Seven, O., Ayan, S. \& Akkaya, E.U. (2017). Amplified chemiluminescence signal for sensing fluoride ions. ACS Omega 2, 3291-3295. DOI: 10.1021/acsomega.7b00537.

10. Shi, X.M., Fan, W.L., Fan, C.H., Lu, Z.L., Bo, Q.B., Wang, Z., Black, C.A., Wang, F.F. \& Wang, Y.Q. (2017). A two-photon fluorescent probe for imaging aqueous fluoride ions in living cells and tissues. Dyes Pigm. 140, 109-115. DOI: 10.1016/j.dyepig.2017.01.038.

11. Li, Y.H., Duan, Y., Zheng, J., Li, J.S., Zhao, W.J., Yang, S. \& Yang, R.H. (2013). SelF-assembly of graphene oxide with a silyl-appended spiropyran dye for rapid and sensitive colorimetric detection of fluoride ions. Anal. Chem. 85, 11456-11463. DOI: $10.1021 / \mathrm{ac} 402592 \mathrm{c}$.

12. Ke, B.W., Chen, W.X., Ni, N.T., Cheng, Y.F., Dai, C.F., Dinh, H. \& Wang, B.H. (2013). A fluorescent probe for rapid aqueous fluoride detection and cell imaging. Chem. Commun. 49, 2494-2496. DOI: 10.1039/C2CC37270C. 
13. Gabrielli, L. \& Mancin, F. (2016). Minimal selF-immolative probe for multimodal fluoride detection. J. Org. Chem. 81, 10715-10720. DOI: 10.1021/acs.joc.6b01787.

14. Kang, Y.F., Qiao, H.X., Meng, L.Y., Xin, Z.H., Ge, L.P., Dai, M.Y., He, Z. \& Zhang, C.H. (2017). Selective fluorescent detection of cysteine over homocysteine and glutathione by a simple and sensitive probe. Aust. J. Chem. 70, 952-956. DOI: 10.1071/CH17208.

15. Li, Z., Xu, Y.Q., Fu, J., Zhu, H.L. \& Qian, Y. (2018). Monitoring of $\mathrm{Au}(\mathrm{III})$ species in plants using a selective fluorescent probe. Chem. Commun. 54, 888-891. DOI: 10.1039/ C7CC08333E.

16. Zheng, X.J., Zhu, W.C., Liu, D., Ai, H., Huang, Y. \& Lu, Z.Y. (2014). Highly selective colorimetric/fluorometric dual-channel fluoride ion probe, and its capability of differentiating cancer cells. ACS Appl. Mater. Interfaces 6, 7996-8000. DOI: 10.1021/am501546h. 